\title{
Fuzzy Rule-Based Classification System for Assessing Coronary Artery Disease
}

\author{
Reza Ali Mohammadpour, ${ }^{1}$ Seyed Mohammad Abedi, ${ }^{2}$ \\ Somayeh Bagheri, ${ }^{3}$ and Ali Ghaemian ${ }^{4}$ \\ ${ }^{1}$ Department of Biostatistics, Faculty of Health, Diabetes Research Center, Mazandaran University of Medical Sciences, \\ Sari 4817844718, Iran \\ ${ }^{2}$ Department of Radiology, Faculty of Medicine, Mazandaran University of Medical Sciences, Sari 4817844718, Iran \\ ${ }^{3}$ Department of Biostatistics, Faculty of Health, Zabol University of Medical Sciences, Zabol, Iran \\ ${ }^{4}$ Mazandaran Heart Center, Mazandaran University of Medical Sciences, Sari 4817844718, Iran
}

Correspondence should be addressed to Reza Ali Mohammadpour; mohammadpour2002@yahoo.com

Received 17 April 2015; Revised 14 July 2015; Accepted 21 July 2015

Academic Editor: Chuangyin Dang

Copyright (C) 2015 Reza Ali Mohammadpour et al. This is an open access article distributed under the Creative Commons Attribution License, which permits unrestricted use, distribution, and reproduction in any medium, provided the original work is properly cited.

\begin{abstract}
The aim of this study was to determine the accuracy of fuzzy rule-based classification that could noninvasively predict CAD based on myocardial perfusion scan test and clinical-epidemiological variables. This was a cross-sectional study in which the characteristics, the results of myocardial perfusion scan (MPS), and coronary artery angiography of 115 patients, 62 (53.9\%) males, in Mazandaran Heart Center in the north of Iran have been collected. We used membership functions for medical variables by reviewing the related literature. To improve the classification performance, we used Ishibuchi et al. and Nozaki et al. methods by adjusting the grade of certainty $C F_{j}$ of each rule. This system includes 144 rules and the antecedent part of all rules has more than one part. The coronary artery disease data used in this paper contained 115 samples. The data was classified into four classes, namely, classes 1 (normal), 2 (stenosis in one single vessel), 3 (stenosis in two vessels), and 4 (stenosis in three vessels) which had 39, 35, 17, and 24 subjects, respectively. The accuracy in the fuzzy classification based on if-then rule was 92.8 percent if classification result was considered based on rule selection by expert, while it was 91.9 when classification result was obtained according to the equation. To increase the classification rate, we deleted the extra rules to reduce the fuzzy rules after introducing the membership functions.
\end{abstract}

\section{Introduction}

In the past years, fuzzy if-then rule-based systems were used basically to control problems, while nowadays they are mainly applied in classification tasks [1-7]. There are many methods for automatically generating and learning the fuzzy if-then rules from numerical data for pattern classification problems $[2,4,5,8,9]$.

The concepts of linguistic statement have been introduced by Zadeh [10] and it is crucial that we see each attribute as linguistic value showed by fuzzy numbers with trapezoidal membership function [11, 12].

After generating the volunteer rule, a set of rules must be chosen to structure the rule based on the classifier. In this paper, we assume a number of prespecified fuzzy sets given by expert's knowledge for each input attribute [13]. The performance of resulting classifiers can be enhanced by using rule weighting [14]. Therefore, our study can have effect on generating, weighting, and selecting rules based on expert opinions or systems outputs.

Therefore, the total number of fuzzy if-then rules generated by partitioning each attribute into $K$ fuzzy subsets in an $n$-dimensional pattern classification is defined as $K^{n}$ $[4,5,15]$. In this work, we adjusted the grade of certainty of fuzzy if-then rules when there was misclassification of an input pattern in an error-correction method [15].

Coronary artery disease (CAD) is the most common cause of mortality in worldwide population and has a high prevalence of mortality rate in both developing and developed countries [16]. Many risk factors such as age, sex, high 
blood pressure, diabetes, obesity, smoking, family history of coronary artery disease, and cholesterol (LDL) have essential role in CAD [17]. Some risk factors such as sex and diabetes are crisp, whereas others are fuzzy sets.

The gold standard method for the diagnosis of CAD is coronary angiography (CA). Since CA is a costly and invasive procedure needing technology and high level of technical experience, it cannot be used to screen large population [1820]. Therefore, noninvasive alternative methods for coronary angiography are necessary. A number of noninvasive CAD diagnosis methods have been proposed in literature [21], namely, exercise stress test and Single Photon Emission Computed Tomography (SPECT) or scintigraphy and Echo. However, the diagnosis accuracy of these tests is not as high as that of coronary angiography. Some studies showed that these medical tests do not have an accurate result and fuzzy set theory can enhance their accuracy [22-25]. Moreover, the diagnosis accuracy of a combination of the results of noninvasive clinical tests and other clinical-epidemiological attributes has been improved by using fuzzy models [26]. Fuzzy systems have been proposed and used to determine the cardiovascular diseases and to assess the risk factors due to the ambiguity and uncertainty of the diagnostic process [27, 28].

Fuzzy rule-based classification system is one of the fuzzy systems. In recent years, fuzzy classifier methods have been commonly used in medical diagnosis. In the literature there are a lot of studies that have worked on the classification of medical data (on cancer and cardiology) using fuzzy classifiers [29, 30]. In 2004, Vig et al. employed fuzzy set theory for the diagnosis of CAD [31]. Allahverdi et al. designed a fuzzy expert system to determine coronary heart disease risk [32] and P. Srivastava and A. Srivastava conducted similar study in India [33] and some of investigators studied improving fuzzy decision systems for CAD diagnosis.

Clinical importance or relevance of features (input variables) in cardiology diagnostic tests can introduce weights for interpretable fuzzy rule selection. CAD diagnosis is a complex and important problem. Some of rules are clinically acceptable and desirable by physicians. In this paper, we added the result of MPS to other attributes for generating fuzzy rules according to physician knowledge and supervised classification with labeled data. Data set is classified using Ishibuchi et al. [34-36] weighted fuzzy rule-based classifier to diagnose CAD and 3 levels of severity of CAD.

The aim of this study was to determine the accuracy of fuzzy rule-based classification that could noninvasively predict the CAD based on myocardial perfusion scan test and clinical-epidemiological variables.

After Introduction in Section 1 we continued, in Section 2, generating, learning, and weighting fuzzy if-then rules. Finally, in Sections 3 and 4, we showed application results and discussion.

\section{Methods: Weighting Fuzzy Classification System}

2.1. Fuzzy If-Then Rule. Let us suppose that our pattern classification problems are $m$ training patterns $X_{p}=\left(X_{p 1}, \ldots\right.$, $\left.X_{p n}\right), p=1, \ldots, m$ with $c$-class $n$-dimensional problem $[5,7,30]$.

First, all attribute values of the given training pattern are transformed in the unit interval $[0,1]$. Thus, the pattern space into the $n$-dimensional pattern classification problem uses unit hypercube $[0,1]^{n}[5,6,34]$. For an $n$-dimensional, $c$-class problem, we apply fuzzy if-then rule of the following form:

$$
\text { Rule } R_{j} \text { : If } x_{1} \text { is } A_{j 1} \text { and } \ldots \text { and } x_{n} \text { is } A_{j n} \text { Then class } C_{j} \text { with } C F_{j} \quad j=1, \ldots, N \text {, }
$$

where $R_{j}$ is the label of the $i$ th fuzzy if-then rule, $N$ is the total number of fuzzy if-then rules, $X=\left[x_{1}, \ldots, x_{n}\right]$ is the pattern vector $n$-dimensional, $A_{j 1}$ presents antecedent fuzzy sets for the $i$ th attribute, $C_{j}$ represent a consequent class (i.e., one of the $c$ classes), and $C F_{j}$ is a certainty grade of the fuzzy if-then $R_{j}[2,5,9,13,15,34,35]$.

As antecedent fuzzy sets we employ trapezoidal fuzzy sets, where we display other partitions of the unit interval into fuzzy sets $[2,36]$.

Using (1), we generate $N$ fuzzy if-then rule that consists of two below steps. The first one is specifying membership function of antecedent fuzzy sets and the second one is determining consequent class $C_{j}$ and certainty grade $C F_{j}$ of the fuzzy rule $R_{j}[2,5,15]$. The antecedent part of the fuzzy if-then rules is initialized manually [2]. For each training pattern, the concept of a weight is applied. The weight of misclassified/rejected patterns is observed as a cost of misclassification or rejection. When a training pattern is misclassified, then the adjustment of fuzzy rules arises. In this study, we can determine both consequent class $C_{j}$ and the grade of certainty $C F_{j}$ for all rules of the following type.

Step 1. Calculate the compatibility grade of training patterns using product as T-norm:

$$
\begin{aligned}
& \mu_{j}\left(X_{p}\right)=\mu_{j 1}\left(x_{p 1}\right) \times \cdots \times \mu_{j n}\left(x_{p n}\right), \\
& \qquad p=1,2, \ldots, m,
\end{aligned}
$$

where $\mu_{j i}(\cdot)$ is the membership function of fuzzy sets $A_{j i}$.

Step 2. For each class $h$ can be calculated $\beta_{\text {class } h}\left(R_{j}\right)$ according to

$$
\beta_{\text {class } h}\left(R_{j}\right)=\sum_{x_{p} \in \text { class } h} \mu_{j}\left(x_{p}\right) \cdot w_{p} \quad h=1,2, \ldots, c .
$$

And $w_{p}$ is the weight of the training pattern. 
Step 3. Find class $\widehat{h}$ that has largest sum of $\beta_{\text {class } h}\left(R_{j}\right)$ :

$$
\beta_{\text {class } \widehat{h}}\left(R_{j}\right)=\max _{1 \leq l \leq c}\left\{\beta_{\text {class } l}\left(R_{j}\right)\right\} .
$$

Note that if two or more classes take the maximum value of (4), then the consequent class $C_{j}$ of the fuzzy rule $R_{j}$ cannot be individually determined, so $C_{j}$ is also specified as $\emptyset$. Let the grade of certainty $C F_{j}$ of fuzzy rule $R_{j}$ be $C F_{j}=0$. If only class takes the maximum value, let $C_{j}$ be class $\widehat{h}$. The grade of certainty $C F_{j}$ can be assigned as follows:

$$
C F_{j}=\frac{\beta_{\text {class } \hat{h}}\left(R_{j}\right)-\bar{\beta}}{\sum_{h} \beta_{\text {class } h}\left(R_{j}\right)} .
$$

With

$$
\bar{\beta}=\frac{\sum_{h \neq \hat{h}} \beta_{\text {classh }}\left(R_{j}\right)}{c-1}
$$

$c$ is the total number of classes.

After generating $N$ fuzzy if-then rules by (1), both the consequent class and the grade of certainty can be determined for all $N$ rules; then a new pattern $X=\left(x_{1}, \ldots, x_{n}\right)$ according to the following procedure can be classified.

Step 1. For class $h(h=1, \ldots, c)$, calculate $\alpha_{\text {class } h}(X)$ as

$$
\alpha_{\text {class } h}(X)=\max \left\{\mu_{j}(X) \cdot C F_{j} \mid C_{j}=h\right\} .
$$

Step 2. Find class $h^{*}$ that has the largest sum of $\alpha_{\text {class } h}(X)$ :

$$
\alpha_{\text {class } h^{*}}(X)=\max _{1 \leq k \leq c}\left\{\alpha_{\text {class } k}(X)\right\}
$$

When multiple classes take the same (maximum) value in (8), then the classification of $X$ cannot be classified (i.e., $X$ is left as an unclassifiable pattern); otherwise, a sign $X$ to class $h^{*}$ (i.e., $X$ ) is considered as a classifiable pattern by (8).

2.2. Learning Fuzzy If-Then Rules. In this section, we use a method for improving classification performance. It adjusts the grade of certainty $C F_{j}$ of each rule in Nozaki et al. [37].

Distributed representation of fuzzy rules is a general concept which is not restricted within the field of pattern classification [38]. The total number of the distributed fuzzy rules can be reduced by removing some unnecessary rules from (1) since such selection of fuzzy rules may require a complicated procedure.

When a fuzzy rule is found, training patterns covered by these rules are removed or their weights are set to zero. Then another fuzzy rule is found and added to the rule set using the modified training set [39].

After generating rules by system, each rule was evaluated by expert for decreasing the rule space dimension. Number of rules inducted by systems was 545 rules which were then reduced to 144 by using expert rule selection. We assigned weights to rules in two cases separately.
When a training pattern was not successfully classified by the fuzzy if-then rule $R_{j}$, its grade of certainty was decreased as

$$
C F_{j}^{\text {new }}=C F_{j}^{\text {old }}-\eta \cdot w_{p} \cdot C F_{j}^{\text {old }} \text {. }
$$

On the contrary, if the training pattern was correctly classified, we could reinforce the grade of certainty in the following manner:

$$
C F_{*}^{\text {new }}=C F_{*}^{\text {old }}+\eta \cdot w_{p} \cdot\left(1-C F_{*}^{\text {old }}\right)
$$

where $\eta$ is a positive constant value. Let $0 \leq \eta \leq 1$, and $w_{p}$ is the weight of the pattern [36].

2.3. Cost Function. This section evaluates the performance of classification systems. Under the hypothesis that a weight is determined to each training pattern, we applied the weight of training patterns as a cost of misclassification. A cost function $\operatorname{cost}(S)$ of a fuzzy classification system $S$ is defined as

$$
\operatorname{Cost}(S)=\sum_{p=1}^{m} w_{p} \cdot z_{p}(S),
$$

where $Z_{p}(S)$ is defined as duplex variable to the classification result of the pattern $x_{p}$ by $S$ : if $x_{p}$ is correctly classified by $S$, then $Z_{p}(S)=0$, and if $x_{p}$ is misclassified or rejected, $Z_{p}(S)=1$ and $m$ is the total number of training patterns. This cost function as good as classification rate is used [36]. We employed four types of weight which were introduced by Nakashima et al. in 2005 [36].

In this paper, we randomly used training pattern $w_{p}$ as $w_{p}=0.25,0.5,0.7,0.9$ and then we randomly assigned a weight to each training pattern. For example, we specified $w_{p}$ as $w_{p}=0.25$ if the training pattern $x_{p}$ belonged to class 1 and $w_{p}=0.25,0.5,0.7,0.9$ if $x_{p}$ belonged to classes 2,3 , and 4 and $w_{p}$ as $w_{p}=0.5$ if the training pattern $x_{p}$ belonged to class 1 and $w_{p}=0.25,0.5,0.7,0.9$ if $x_{p}$ belonged to classes 2,3 , and 4 , and so forth.

\section{Application Results}

3.1. Patients. This study was a cross-sectional study in which characteristics, results of myocardial perfusion scan (MPS), and coronary artery angiography of 115 patients, 62 (53.9\% male), in Mazandaran Heart Center (Iran) have been collected. The coronary artery disease data used in this paper contained 115 samples classified into four classes, namely, classes 1 (normal), 2 (stenosis in one single vessel), 3 (stenosis in two vessels), and 4 (stenosis in three vessels) having 39, 35,17 , and 24 subjects, respectively. The dataset included ten input variables (age, sex, diabetes, cholesterol level, triglyceride level, low density lipoprotein (LDL), systolic blood pressure, summed stress score (SSS), smoking, and genetic factor) and one output. We used membership functions for medical variables according to literature review. For these variables we took 3 fuzzy sets for age (young age, middle age, and old age), 3 fuzzy sets for the cholesterol level and triglyceride level (normal, borderline, and high), 3 fuzzy sets 

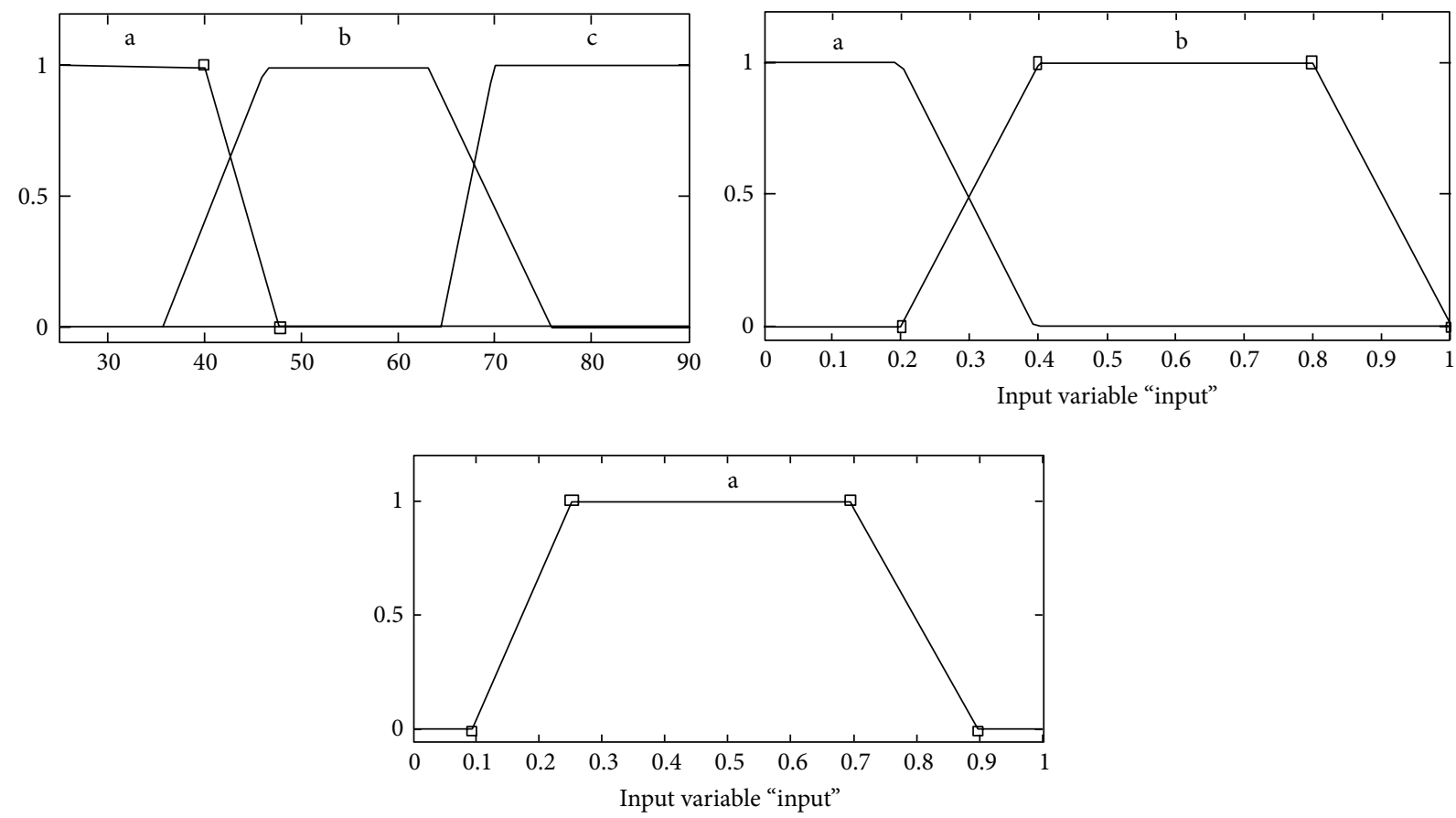

FIgURE 1: Membership function.

for LDL (normal, middle, and high), 4 fuzzy sets for systolic blood pressure (low, middle, high, and very high), and 4 fuzzy sets for SSS (normal, mild, moderate, and severe).

Sex had two stages $(0=$ male and $1=$ female $)$, smoking had two parts $(0=$ nonsmoking and $1=$ smoking $)$, and genetic factor diabetes had two sections $(0=$ no and $1=$ yes $)$.

The type of membership functions for each fuzzy set applied was the trapezoidal membership function as shown in Figure 1 and membership functions of input variables were defined by form in Table 1.

3.2. Fuzzy Rule Base. The main part in fuzzy system is the rule base and the results in a fuzzy classification system depend on the fuzzy rules. This system includes 144 rules. Antecedent part of all rules has more than one part. In this study, we used linguistic variables including middle $(M)$, high $(H)$, very high $(\mathrm{VH})$, low $(\mathrm{L})$, young $(\mathrm{Y})$, old $(\mathrm{O})$, moderate $(\mathrm{Mo})$, mild $(\mathrm{Mi})$, severe (S), normal (NR), borderline (B), middle age $(\mathrm{M})$, middle (mid), yes $(\mathrm{Y})$, and no $(\mathrm{N})$ for input variables.

The units of the used input variables were age, cholesterol (CL), triglyceride (TG), systolic blood pressure (SY), LDL, SSS, sex, diabetes (DI), genetic factor (GF), and smoking (SM).

Parts of the fuzzy rules are shown in Table 2. A series of 144 rules are formed.

After learning fuzzy if-then rules by training patterns, we used different weights and constants values. Correct classification rates were decreased by assigning different weights to rules and the highest classification rate was for $w_{p}=$ 0.25 for all classes. The results of correct classification rates are displayed in Table 3. After generating rules by system, classification rate was $92.4 \%$ by 545 rules which were then reduced to 144 by using expert rule selection; accuracy rate has computed $92.8 \%$.

Multiple logistic regression (MLR) was used for detecting the risk factors effects on CAD (presence or absence). Sensitivity and specificity of MLR were computed, $88.15 \%$ and $64.1 \%$, respectively. Also, clinical test PMS in comparison to angiography was found to have sensitivity of $84 \%$ and specificity of $92 \%$ higher than MLR model.

All patients with CAD by angiography were detected by this proposed fuzzy rule-based classification $(\mathrm{Se}=100 \%)$ and overall accuracy was $92.8 \%$.

\section{Discussion and Conclusion}

The most important result of this study is the classification of coronary artery disease (CAD) with a high accuracy. Correct classification rate depends on the number of input variables or characteristics, type, and combination of fuzzy rules. We used membership functions for medical variables by reviewing the related literature. These membership functions lead to a high quality of precision in many fields of medicine. We used fuzzy inference method with combination of weighted rules by expert and supervisor pattern classification. This is similar to Chen and Chang study with high accuracy rate (96.88\%) in classification [40]. Chen and Fang studied a fuzzy classification method for Iris data with $96.72 \%$ accuracy classification rate [41]. They generated fuzzy weighted rules for Iris data with genetic algorithm [42]. The study conducted by Allahverdi et al. for determining the risk factors for CAD had a high corrected classification rate [32]. 
TABLE 1: Membership functions for attributes of CAD.

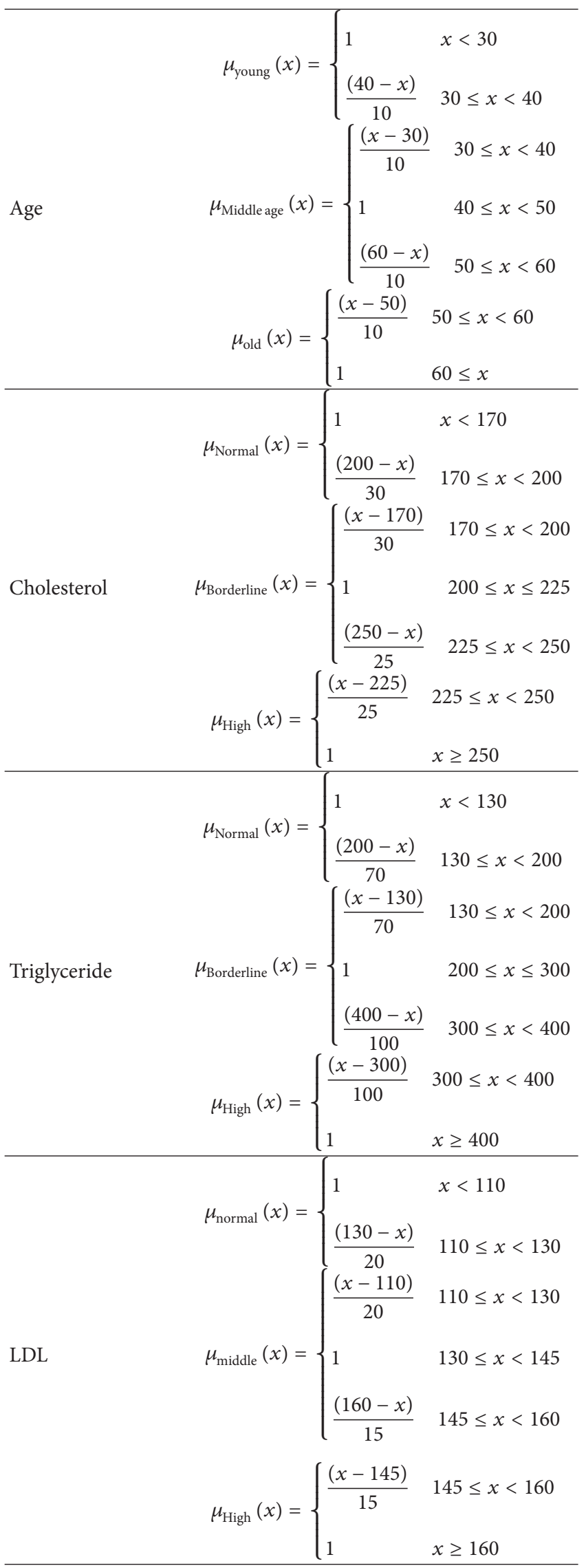

TABLE 1: Continued.

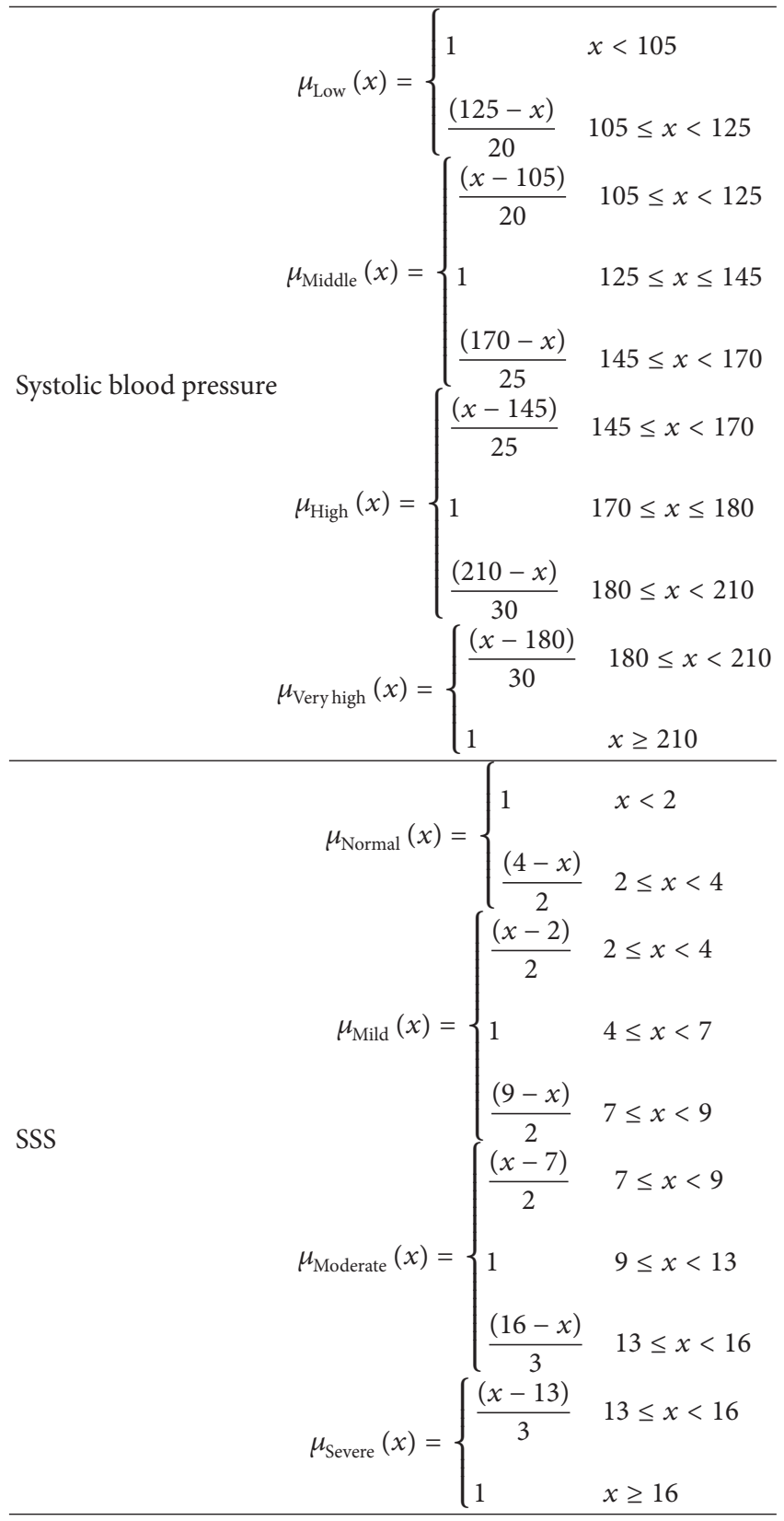

Vig et al. [31], P. Srivastava and A. Srivastava [33] and Kaya et al. [43] also studied fuzzy set and systems for CAD, cardiac analysis, and congenital heart disease by using fuzzy expert systems.

This study showed that sensitivity and specificity of PMS compared with angiography were acceptable by MLR, but when it was combined with other clinical-epidemiological variables, fuzzy rule-based classification model improved classification rate.

According to the findings of this study, interpretable fuzzy rule-based classification can determine the most important risk factors for $\mathrm{CAD}$ and correctly detect the patients who do 
TABLE 2: Sample fuzzy rules.

\begin{tabular}{|c|c|c|c|c|c|c|c|c|c|c|c|c|}
\hline \multirow{2}{*}{ Rule number } & \multicolumn{10}{|c|}{ If } & \multicolumn{2}{|c|}{ Then } \\
\hline & Age & $\mathrm{CL}$ & TG & LDL & SY & SSS & Sex & DI & SM & GF & Heart disease & $C F_{j}$ \\
\hline 1 & $\mathrm{M}$ & NR & NR & NR & $\mathrm{L}$ & Mo & $\mathrm{F}$ & $\mathrm{N}$ & $\mathrm{Y}$ & $\mathrm{N}$ & NR & 1.000 \\
\hline 2 & M & $\mathrm{B}$ & $\mathrm{B}$ & Mid & $\mathrm{L}$ & NR & $\mathrm{F}$ & $\mathrm{N}$ & $\mathrm{N}$ & $\mathrm{Y}$ & NR & 1.000 \\
\hline 3 & M & B & B & Mid & Mid & NR & $\mathrm{F}$ & $\mathrm{N}$ & $\mathrm{Y}$ & $\mathrm{Y}$ & NR & 1.000 \\
\hline 4 & $\mathrm{O}$ & B & B & Mid & Mid & $\mathrm{Mi}$ & $\mathrm{F}$ & $\mathrm{N}$ & $\mathrm{N}$ & $\mathrm{N}$ & NR & 0.941 \\
\hline 5 & M & B & B & NR & $\mathrm{H}$ & Mo & $\mathrm{F}$ & $\mathrm{Y}$ & $\mathrm{N}$ & $\mathrm{Y}$ & NR & 0.955 \\
\hline 6 & M & B & B & Mid & Mid & Mo & $\mathrm{F}$ & $\mathrm{N}$ & $\mathrm{N}$ & $\mathrm{Y}$ & 3.v & 1.000 \\
\hline 7 & $\mathrm{O}$ & B & B & Mid & $\mathrm{L}$ & S & $\mathrm{F}$ & $\mathrm{N}$ & $\mathrm{N}$ & $\mathrm{N}$ & S.v & 0.642 \\
\hline 8 & M & B & $\mathrm{B}$ & Mid & Mid & $\mathrm{Mi}$ & $\mathrm{F}$ & $\mathrm{Y}$ & $\mathrm{Y}$ & $\mathrm{Y}$ & S.V & 0.989 \\
\hline 9 & M & B & NR & Mid & Mid & $\mathrm{Mi}$ & M & $\mathrm{N}$ & $\mathrm{Y}$ & $\mathrm{Y}$ & S.V & 1.000 \\
\hline 10 & $\mathrm{O}$ & B & $\mathrm{B}$ & Mid & $\mathrm{H}$ & $S$ & $\mathrm{M}$ & $\mathrm{Y}$ & $\mathrm{N}$ & $\mathrm{N}$ & $3 . v$ & 1.000 \\
\hline 11 & M & B & NR & NR & $\mathrm{L}$ & S & M & $\mathrm{N}$ & $\mathrm{N}$ & $\mathrm{N}$ & 2.v & 1.000 \\
\hline 12 & M & B & $\mathrm{B}$ & NR & Mid & $S$ & M & $\mathrm{Y}$ & $\mathrm{Y}$ & $\mathrm{Y}$ & $2 . \mathrm{v}$ & 1.000 \\
\hline 13 & $\mathrm{O}$ & $\mathrm{H}$ & B & NR & Mid & Mo & M & $\mathrm{N}$ & $\mathrm{Y}$ & $\mathrm{Y}$ & S.V & 0.795 \\
\hline 14 & M & NR & NR & NR & $\mathrm{H}$ & S & M & $\mathrm{Y}$ & $\mathrm{Y}$ & $\mathrm{N}$ & $3 . \mathrm{V}$ & 1.000 \\
\hline$\vdots$ & & & & & $\vdots$ & & & & & & & $\vdots$ \\
\hline
\end{tabular}

TABLE 3: Results of correct classification rates with different weighting rules.

\begin{tabular}{|c|c|c|c|}
\hline$\eta$ & $w_{p}$ for all classes & $\operatorname{Cost}(S)$ & Classification rate \\
\hline$\eta=0.1$ & ( $w_{p}=0.25$ for all classes $)$ & 8.5 & $92.8 \%$ \\
\hline$\eta=0.3$ & $\left(w_{p}=0.25\right.$ for all classes $)$ & 9.8 & $91.2 \%$ \\
\hline$\eta=0.1$ & $\begin{array}{l}\left(x_{p} \in \text { class } 1 w_{p}=0.5\right. \\
x_{p} \in \text { class } 2 w_{p}=0.25 \\
x_{p} \in \text { class } 3 w_{p}=0.5 \\
\left.x_{p} \in \text { class } 4 w_{p}=0.9\right)\end{array}$ & 12.4 & $89.3 \%$ \\
\hline$\eta=0.3$ & $\begin{array}{l}\left(x_{p} \in \text { class } 1 w_{p}=0.5\right. \\
x_{p} \in \text { class } 2 w_{p}=0.25 \\
x_{p} \in \text { class } 3 w_{p}=0.5 \\
\left.x_{p} \in \text { class } 4 w_{p}=0.9\right)\end{array}$ & 19.8 & $83.6 \%$ \\
\hline$\eta=0.1$ & $\begin{array}{l}\left(x_{p} \in \text { class } 1 w_{p}=0.7\right. \\
x_{p} \in \text { class } 2 w_{p}=0.7 \\
x_{p} \in \text { class } 3 w_{p}=0.5 \\
\left.x_{p} \in \text { class } 4 w_{p}=0.25\right)\end{array}$ & 15.6 & $88.9 \%$ \\
\hline$\eta=0.3$ & $\begin{array}{l}\left(x_{p} \in \text { class } 1 w_{p}=0.7\right. \\
x_{p} \in \text { class } 2 w_{p}=0.7 \\
x_{p} \in \text { class } 3 w_{p}=0.5 \\
\left.x_{p} \in \text { class } 4 w_{p}=0.25\right)\end{array}$ & 18.9 & $86.4 \%$ \\
\hline$\eta=0.1$ & $\begin{array}{l}\left(x_{p} \in \text { class } 1 w_{p}=0.9\right. \\
x_{p} \in \text { class } 2 w_{p}=0.7 \\
x_{p} \in \text { class } 3 w_{p}=0.7 \\
\left.x_{p} \in \text { class } 4 w_{p}=0.5\right)\end{array}$ & 26.2 & $73.1 \%$ \\
\hline$\eta=0.3$ & $\begin{array}{l}\left(x_{p} \in \text { class } 1 w_{p}=0.9\right. \\
x_{p} \in \text { class } 2 w_{p}=0.7, \\
x_{p} \in \text { class } 3 w_{p}=0.7 \\
\left.x_{p} \in \text { class } 4 w_{p}=0.5\right)\end{array}$ & 30.1 & $71.8 \%$ \\
\hline
\end{tabular}

not need invasive tests such as coronary artery angiography and have a high classification accuracy rate.

\section{Conflict of Interests}

The authors declare that there is no conflict of interests regarding the publication of this paper.

\section{Acknowledgments}

The authors acknowledge Ali Adeli for supporting them in using MATLAB programming. They are also grateful to all who helped them in data collection procedure, particularly, patients. This paper is part of a M.S. thesis by S. Bagheri and has used a grant of Deputy 
of Research in Mazandaran University of Medical Sciences.

\section{References}

[1] M. S. Aabdeh, J. Habibi, and E. Soroush, "Induction of fuzzy classification systems via evolutionary ACO-BASED algorithms," International Journal of Simulation, Systems, Science and Technology, vol. 9, no. 3, pp. 1-8, 2008.

[2] T. Nakashima, G. Schaefer, Y. Yokota, and H. Ishibuchi, "A weighted fuzzy classifier and its application to image processing tasks," Fuzzy Sets and Systems, vol. 158, no. 3, pp. 284-294, 2007.

[3] J. A. Roubos, M. Setnes, and J. Abonyi, "Learning fuzzy classification rules from labeled data," Information Sciences, vol. 150, no. 1-2, pp. 77-93, 2003.

[4] E. G. Mansoori, M. J. Zolghadri, and S. D. Katebi, "Using distribution of data to enhance performance of fuzzy classification systems," Iranian Journal of Fuzzy Systems, vol. 4, no. 1, pp. 2136, 2007.

[5] T. Nakashima, Y. Yokota, H. Ishibuchi, and A. Bargiela, "Constructing fuzzy classification systems from weighted training patterns," in Proceedings of the IEEE International Conference on Systems, Man and Cybernetics, vol. 3, pp. 2386-2391, IEEE, October 2004.

[6] H. Mohamadi, J. Habibi, M. S. Abadeh, and H. Saadi, "Data mining with a simulated annealing based fuzzy classification system," Pattern Recognition, vol. 41, no. 5, pp. 1841-1850, 2008.

[7] F. Hoffmann, "Combining boosting and evolutionary algorithms for learning of fuzzy classification rules," Fuzzy Sets and Systems, vol. 141, no. 1, pp. 47-58, 2004.

[8] A. Lekova, L. Mikhailov, D. Boyadjiev, and A. Nabout, "Redundant fuzzy rules exclusion by genetic algorithms," Fuzzy Sets and Systems, vol. 100, no. 1-3, pp. 235-243, 1998.

[9] H. Ishibuchi, T. Murata, and M. Gen, "Performance evaluation of fuzzy rule-based classification systems obtained by multiobjective genetic algorithms," Computers and Industrial Engineering, vol. 35, no. 3-4, pp. 575-578, 1998.

[10] L. A. Zadeh, "Fuzzy sets," Information and Computation, vol. 8, pp. 338-353, 1965.

[11] Y.-C. Hu, R.-S. Chen, and G.-H. Tzeng, "Finding fuzzy classification rules using data mining techniques," Pattern Recognition Letters, vol. 24, no. 1-3, pp. 509-519, 2003.

[12] Y.-C. Hu, "Sugeno fuzzy integral for finding fuzzy if-then classification rules," Applied Mathematics and Computation, vol. 185, no. 1, pp. 72-83, 2007.

[13] M. Z. Jahromi and M. Taheri, "A proposed method for learning rule weights in fuzzy rule-based classification systems," Fuzzy Sets and Systems, vol. 159, no. 4, pp. 449-459, 2008.

[14] E. G. Mansoori, M. J. Zolghadri, and S. D. Katebi, "A weighting function for improving fuzzy classification systems performance," Fuzzy Sets and Systems, vol. 158, no. 5, pp. 583-591, 2007.

[15] T. Nakashima, G. Nakai, and H. Ishibuchi, "Improving the performance of fuzzy classification systems by membership function learning and feature selection," in Proceedings of the IEEE International Conference on Fuzzy Systems (FUZZ-IEEE '02), vol. 1, pp. 488-493, Honolulu, Hawaii, USA, May 2002.

[16] R. J. Goldberg, D. Goff, L. S. Cooper, R. Luepker, J. Zapka, and V. Bittner, "Age and sex differences in presentation of symptoms among patients with acute coronary disease: the REACT trial," Coronary Artery Disease, vol. 11, no. 5, pp. 399-407, 2000.
[17] Z. N. Hatmi, S. Tahvildari, A. Gafarzadeh Motlag, and A. Sabouri Kashani, "Prevalence of coronary artery disease risk factors in Iran: a population based survey," BMC Cardiovascular Disorders, vol. 7, article 32, 2007.

[18] D. Pal, K. M. Mandana, S. Pal, D. Sarkar, and C. Chakraborty, "Fuzzy expert system approach for coronary artery disease screening using clinical parameters," Knowledge-Based Systems, vol. 36, pp. 162-174, 2012.

[19] E. P. Ephzibah and V. Sundarapandian, "A neuro-fuzzy expert system for heart disease diagnosis," Computer Science \& Engineering, vol. 2, no. 1, pp. 17-23, 2012.

[20] A. Lahsasna, R. N. Ainon, R. Zainuddin, and A. Bulgiba, "Design of a fuzzy-based decision support system for coronary heart disease diagnosis," Journal of Medical Systems, vol. 36, no. 5, pp. 3293-3306, 2012.

[21] H. R. Marateb and S. A. Goudarzi, "A noninvasive method for coronary artery diseases diagnosis using a clinicallyinterpretable fuzzy rule-based system," Journal of Research in Medical Sciences, vol. 20, pp. 214-223, 2015.

[22] R. M. Peters, S. A. Shanies, and J. C. Peters, "Predicting extent of coronary disease: fuzzy cluster analysis vs. Duke treadmill score," Journal of Clinical and Basic Cardiology, vol. 3, no. 1, pp. 39-41, 2000.

[23] R. A. Mohammadpour, G. Babaee, M. Mahmodi, A. Memariani, and A. Mohseni, "Evaluation of exercise test variables in diagnosis and prediction of the severity of coronary artery disease by fuzzy discriminant analysis," Journal of Mazandaran University of Medical Sciences, vol. 11, no. 33, 2002 (Persian).

[24] H. F. Pop, T. L. Pop, and C. Sarbu, "Assessment of heart disease using fuzzy classification techniques," The Scientific World Journal, vol. 1, pp. 369-390, 2001.

[25] F. Cachin, J. Lipiecki, D. Mestas et al., "Preliminary evaluation of a fuzzy logic-based automatic quantitative analysis in myocardial SPECT,' Journal of Nuclear Medicine, vol. 44, no. 10, pp. 1625-1632, 2003.

[26] R. M. Peters, S. A. Shanies, and J. C. Peters, "Fuzzy cluster analysis off positive stress tests, a new method of combining exercise test variables to predict extent of coronary artery disease," The American Journal of Cardiology, vol. 76, no. 10, pp. 648-651, 1995.

[27] D. Kalmanson and H. F. Stegall, "Cardiovascular investigations and fuzzy sets theory," The American Journal of Cardiology, vol. 35, no. 1, pp. 80-84, 1975.

[28] K.-P. Adlassnig, "Fuzzy set theory in medical diagnosis," IEEE Transactions on Systems, Man and Cybernetics, vol. 16, no. 2, pp. 260-265, 1986.

[29] R. Jain, J. Mazumdar, and W. Moran, "Application of fuzzyclassifier system to coronary artery disease and breast cancer," Australasian Physical and Engineering Sciences in Medicine, vol. 21, no. 3, pp. 141-147, 1998.

[30] R. Jain and A. Abraham, "A comparative study of fuzzy classification methods on breast cancer data," Australasian Physical and Engineering Sciences in Medicine, vol. 27, no. 4, pp. 213-218, 2004.

[31] R. Vig, N. M. Handa, and H. K. Bali Sridhar, "Fuzzy diagnostic system for coronary artery disease," IE(I) Journal-ID, vol. 85, pp. 41-46, 2004.

[32] N. Allahverdi, S. Torun, and I. Saritas, "Design of a fuzzy expert system for determination of coronary heart disease risk," in Proceedings of the International Conference on Computer Systems and Technologies (CompSysTech '07), ACM, June 2007. 
[33] P. Srivastava and A. Srivastava, "A note on soft computing approach for cardiac analysis," Journal of Basic and Applied Scientific Research, vol. 2, no. 1, pp. 376-385, 2012.

[34] H. Ishibuchi and T. Yamamoto, "Comparison of heuristic rule weight specification methods," in Proceedings of the IEEE International Conference on Fuzzy Systems (FUZZ-IEEE '02), vol. 2, pp. 908-913, IEEE, Honolulu, Hawaii, USA, May 2002.

[35] T. Nakashima, Y. Yokota, H. Ishibuchi, G. Schaefer, A. Drastich, and M. Zavisek, "Constructing cost-sensitive fuzzy-rulebased systems for pattern classification problems," Journal of Advanced Computational Intelligence and Intelligent Informatics, vol. 11, no. 6, pp. 546-553, 2007.

[36] T. Nakashima, Y. Yokota, and H. Ishibuchi, "Learning fuzzy if-then rules for pattern classification with weighted training patterns," in Proceedings of the 4th Conference of the European Society for Fuzzy Logic and Technology (EUSFLAT-LFA '05), pp. 1064-1069, 2005.

[37] K. Nozaki, H. Ishibuchi, and H. Tanaka, "Adaptive fuzzy rule-based classification systems," IEEE Transactions on Fuzzy Systems, vol. 4, no. 3, pp. 238-250, 1996.

[38] H. Ishibuchi, K. Nozaki, and H. Tanaka, "Distributed representation of fuzzy rules and its application to pattern classification," Fuzzy Sets and Systems, vol. 52, no. 1, pp. 21-32, 1992.

[39] M. Eftekhari, M. J. Zolghadri, and S. D. Katebi, "New criteria for rule selection in fuzzy learning classifier systems," Iranian Journal of Fuzzy Systems, vol. 3, no. 1, pp. 77-89, 2006.

[40] S.-M. Chen and C.-H. Chang, "A new method to construct membership functions and generate weighted fuzzy rules from training instances," Cybernetics and Systems, vol. 36, no. 4, pp. 397-414, 2005.

[41] S. M. Chen and Y. D. Fang, "A new method to deal with fuzzy classification problems by tuning membership functions for fuzzy classification systems," Journal of the Chinese Institute of Engineers, vol. 28, no. 1, pp. 169-173, 2005.

[42] S.-M. Chen and H.-L. Lin, "Generating weighted fuzzy rules from training instances using genetic algorithms to handle the iris data classification problem," Journal of Information Science and Engineering, vol. 22, no. 1, pp. 175-188, 2006.

[43] E. Kaya, B. Oran, and A. Arslan, "A diagnostic fuzzy rule-based system for congenital heart disease," World Academy of Science, Engineering and Technology, vol. 78, pp. 253-256, 2011. 


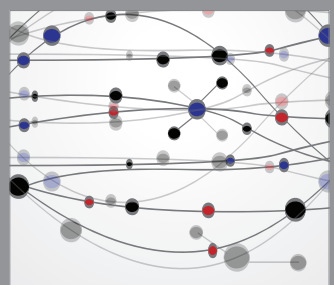

The Scientific World Journal
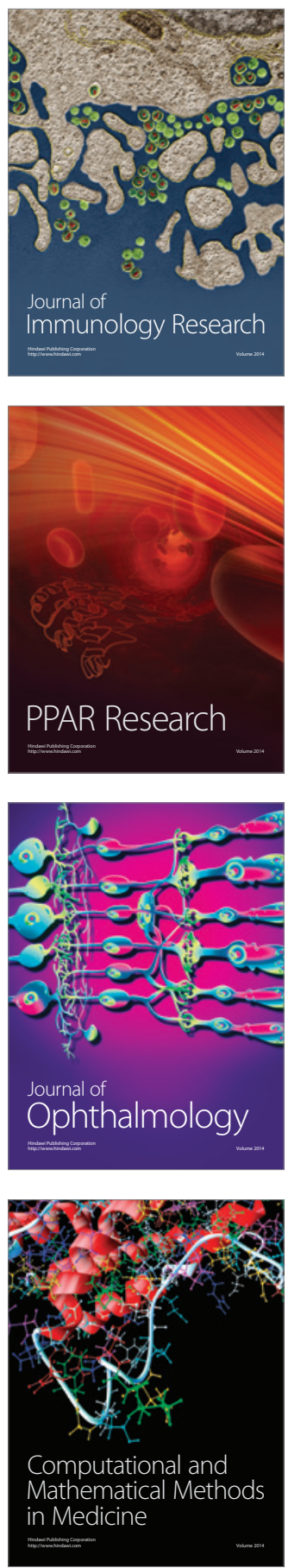

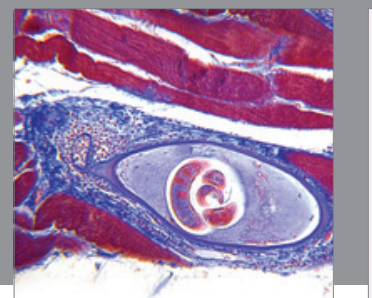

Gastroenterology

Research and Practice
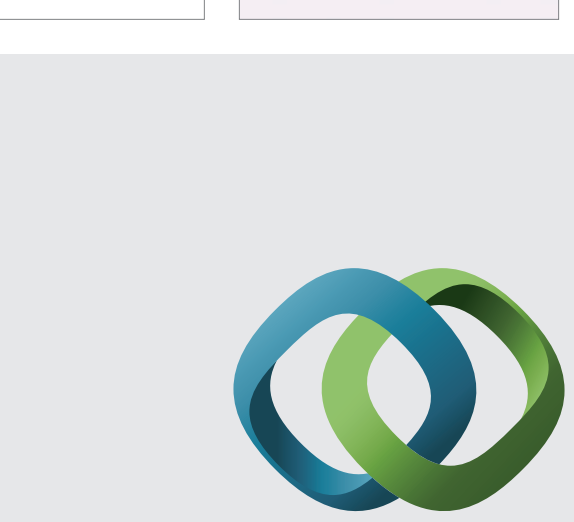

\section{Hindawi}

Submit your manuscripts at

http://www.hindawi.com
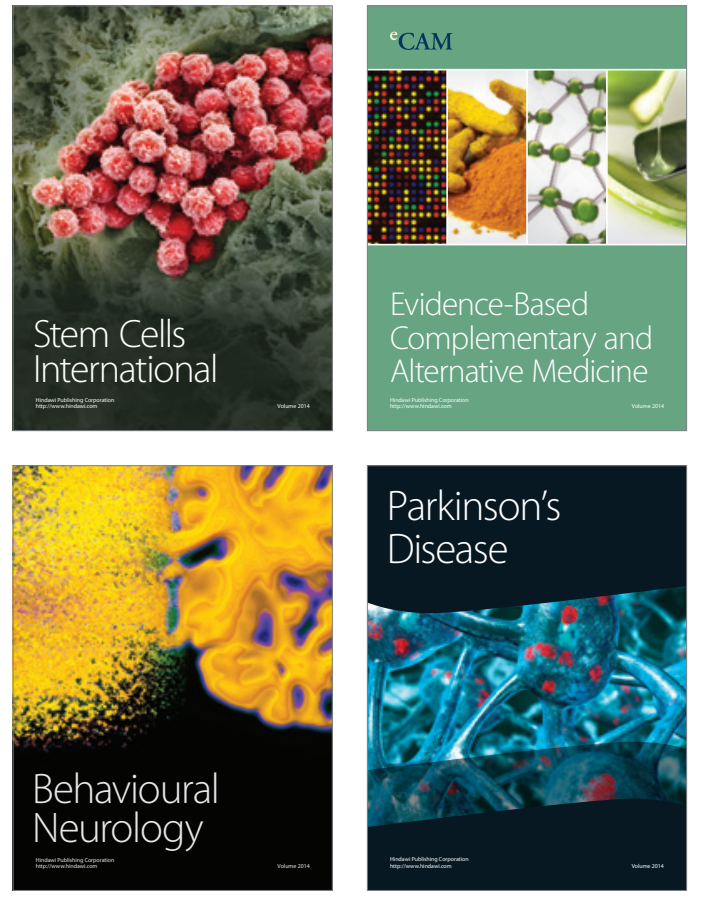
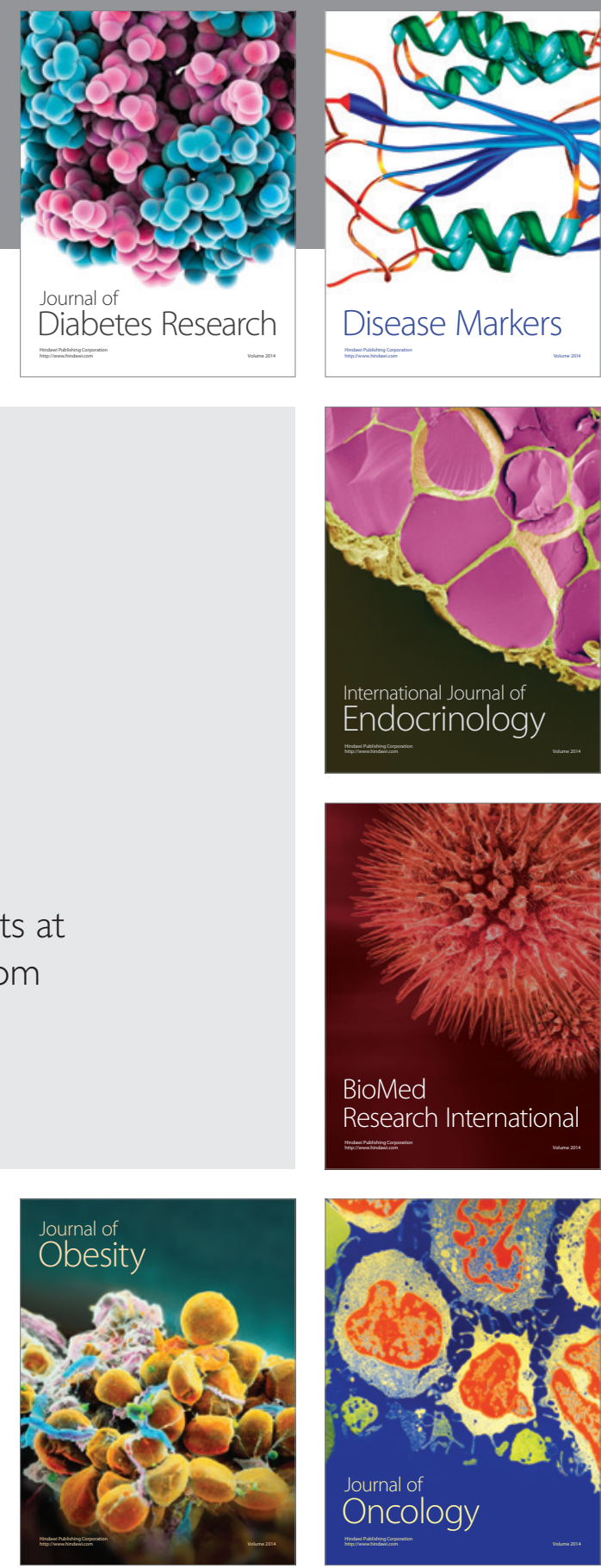

Disease Markers
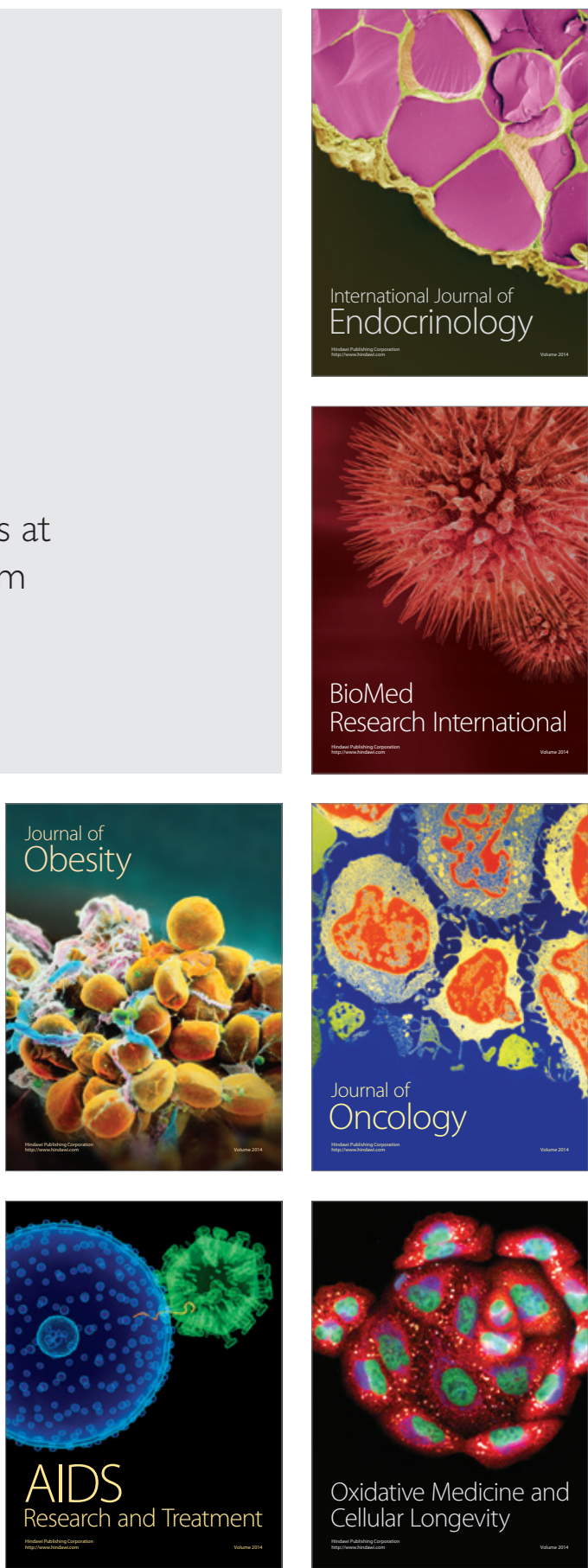